\title{
Anti-wrinkle Effect of a Palmitoyl Oligopeptide Complex on Human Keratinocytes and Fibroblasts Through TGF- $\beta 1$ Pathway
}

\author{
Yina Lu', Guoxun Wang ${ }^{1}$, Dong Liu ${ }^{2}$, Jun Tian ${ }^{1, ~ * ~}$ \\ ${ }^{1}$ Shanghai JAKA Biotech Co. Ltd, Shanghai, China \\ ${ }^{2}$ Suzhou Greenleaf Daily Commodity Co., Ltd., Suzhou, China
}

Email address:

inna@greaf.com.cn (Yina Lu), tianjun@greaf.com.cn (Jun Tian)

${ }^{*}$ Corresponding author

To cite this article:

Yina Lu, Guoxun Wang, Dong Liu, Jun Tian. Anti-wrinkle Effect of a Palmitoyl Oligopeptide Complex on Human Keratinocytes and Fibroblasts Through TGF- $\beta 1$ Pathway. Cell Biology. Vol. 8, No. 2, 2020, pp. 33-38. doi: 10.11648/j.cb.20200802.13

Received: October 29, 2020; Accepted: November 9, 2020; Published: November 19, 2020

\begin{abstract}
Many researchers revealed that dermal fibroblast which mainly synthesis the collagen and elastin in dermis were affects by skin ageing, such as the proliferation rate and gene expression level. Oligopeptides were verified by many reports on anti-aging focus on collagen synthesis, but few literatures revealed the molecular mechanism of oligopeptides on skin aging. The potential anti-ageing effect and the mechanism of PTC, a new palmitoyl oligopeptide complex, were evaluated. MTT assay was adopted to detect the anti-aging effect of PTC in both human dermal fibroblast HDF-a and keratinocyte NHEK. The mechanism of PTC was detected further by full transcriptome analysis using RNA-sequencing method, and ELISA analysis of key proteins. Skin wrinkles were measured by VC98 in vivo. PTC showed positive effect in cell proliferation and the biosynthesis of collagens, intergrins not only on fibroblasts, but also on keratinocytes. Moreover, many pathways were confirmed in the anti-aging effect of PTC, such as TGF- $\beta 1$ pathway and ECM-receptor interaction. A cream contained $5.0 \%$ PTC showed significant improvement of skin wrinkles and roughness. So that, PTC has a potentially beneficial effect on the cell proliferation, the biosynthesis of ECM related proteins and the expression of many genes which are involved in cell cycle and secretory features to slow down skin aging. The molecular pathway maybe targets the TGF- $\beta 1$ in both epidermis and dermis.
\end{abstract}

Keywords: Anti-wrinkle, A Palmitoyl Oligopeptide Complex, Transcriptome, TGF- $\beta 1$

\section{Introduction}

Skin is a multifunctional organ but, alongside every other organ system, is subject to both intrinsic (chronological) and extrinsic (environmental) aging. Skin function is mediated primarily by the structure of the epidermal and dermal layers. The epidermis as well as the dermis is becoming thinner and the dermal epidermal junction flattens in skin aging [1]. Many researchers revealed that dermal fibroblast which mainly synthesis the collagen and elastin contribute to maintaining the skin's elasticity and inhibit wrinkling of skin in vitro and in vivo [2-4], and many ingredient such as plants and peptides were improved have functions on maintaining skin elasticity, strengthening joints and retaining moisture [5, $6]$, especially the collagen and elastin. Due to the large size of the collagen molecule unable to absorb, oligopeptides were been widely used recently, especially the palmitoyl oligopeptides, including tripeptide to six peptide.

Oligopeptides were verified by many reports on anti-aging focus on collagen synthesis [5-7]. During aging, the dermis undergoes progressive fragmentation that impairs fibroblast attachment with consequent reduction of size. In aged skin, reduced size of fibroblasts is accompanied by their decreased production of key extracellular matrix (ECM) components, such as type I collagen, fibronectin, connective tissue growth factor (CTGF) and transform growth factor (TGF- $\beta$ ) $[8,9]$. Interestingly, many of the dermal ECM-related genes that are downregulated in aged human skin are regulated by the 
TGF- $\beta$ pathway [9]. And many other pathways are also involved in skin aging, such as IGF-FOXO pathway, MAPK pathway, rapamycin (mTOR) pathway [10-12]. There are few literatures revealing the molecular mechanism of oligopeptides on skin aging.

We combined two high throughputs screening methods focus on cell proliferation and type collagen I synthesis which is the common factors related to aging, to validate lots of active ingredients from different plants and peptides, and finally got a palmitoyl oligopeptide complex PTC, which consists of hydrolyzed collagen and oligopeptide. Then the anti-aging effect of PTC was identified in both fibroblasts and keratinocytes, and finally verified in human skin. The mechanism of PTC against skin aging was confirmed by transcriptome (RNA-seq) in human fibroblasts, and ELISA analysis confirmed the expressions of key proteins involved in TGF- $\beta 1$ pathways.

\section{Materials and Methods}

\subsection{PTC Preparation}

The palmitoyl oligopeptides (ZPC peptide, China) were mixed with different proportions and dissolved in 1 , 2-hexanediol (CAS No. 6920-22-5), followed by hydrolyzed collagen added into the liquid. Finally, PTC solution was obtained through filtration.

\subsection{Cell Proliferation Experiments}

Human dermal fibroblast from adult (HDF-a) and normal human epidermal keratinocytes (NHEK) were used to detect the proliferation effect according to literatures [13, 14]. Briefly, Cells were seeded in the 96-well plate at a density of 5000 cells/well. When cultured overnight in FM or DMEM media with $10 \% \mathrm{FBS}$, the cells were continually incubated with various concentrations of PTC (0.004- $1 \%)$ in the medium without FBS for 24 to $72 \mathrm{hrs}$ respectively. Cells treated with vehicle served as a control. The cell proliferation rate was estimated by MTT (Sigma, USA) assay at $492 \mathrm{~nm}$ after incubation using the Multiscan FC microplate reader (Thermo Fisher Scientific, USA). From the values obtained, the percentage proliferation was calculated as in

$$
\text { Viability (\%) }=\text { OD } 492_{\text {treatment }} / \mathrm{OD} 492_{\text {control }} \times 100 \%
$$

\subsection{Transcriptome Sequencing}

Twenty-four hours after the PTC treated $(0.5 \%$ and $1 \%$ concentration) in FM medium without FBS, the purifid total RNA of the human fibroblast was obtained with Trizol (Thermo Fisher Scientific). The RNA (400 ng) was converted to the cDNA library by a TruSeq Stranded LT mRNA kit (Illumina, San Diego, CA, USA) [15-17]. Specific adapters for Illumina universal and qPCR were used to quantify the cDNA libraries. One unique identified sequence was added for sample separation after sequencing each library, thus allowing sequencing of all samples during the same run and minimizing technical variations. Libraries were sequenced on a HiSeq
2500 platform (Illumina) in the High Output mode.

\subsection{Detection of Collagens}

The synthesis of type I and III collagen were detected on HDF-a cells by immunofluorescent assay [18, 19]. HDF-a cells were treated with PTC (0.004-1\%) similar with proliferation assay. For visualization of type I and III collagens, PTC treated HDF-a cells were firstly fixed with cold methanol (CAS No. 67-56-1; Sinopharm, China) at $4{ }^{\circ} \mathrm{C}$ for $10 \mathrm{~min}$, and then $0.5 \%$ triton X-100 (Sigma, USA) was incubated with cells for $15 \mathrm{~min}$. After washing, cells were blocked with $3 \%$ BSA for $1.5 \mathrm{hrs}$ at room temperature. Finally cells were stained with anti-collagen I and III antibody (Abcam, CA) at $4^{\circ} \mathrm{C}$ overnight and anti-rabbit IgG Alexa Fluor (R) 488 antibody (CST, USA) was used to detect the fluorescence. The nuclei were stained with DAPI (Abcam, $\mathrm{CA}$ ) and the images were captured using fluorescence microscope (Leica, GER).

\subsection{ELISA Detection}

The DEJ composition proteins such as laminin 5, intergrin $\beta 1$, fibronectin and four type collagens were detected on NHEK and HDF-a cells by ELISA assay [19-21]. After incubation period, the supernatant and cells were collected and estimated using commercially available ELISA kits (Elabscience, China) according to its manual. The percentage rate of growth was calculated as in

$$
\text { Growth rate }(\%)=\mathrm{OD} 450_{\text {treatment }} / \mathrm{OD} 450_{\text {control }} \times 100 \%
$$

\subsection{Skin Wrinkles Evaluation}

The in vivo study was performed as a double blinded, randomized and placebo controlled study to follow the internationally recognized ethical principles. A clinical study of 30 subjects (the average age was 41.5) was performed to evaluate the effects of PTC on facial wrinkles and roughness $[22,23]$. During 8 weeks treatment, participants applied 2 $\mathrm{mg} / \mathrm{cm}^{2}$ test formula (contained 5\% PTC) and vehicle around the left and right eye every morning and evening after cleansing. Photo were taken and analyzed by visioscan VC98 (CK company, GER) using SELS multi parameter analysis at the following time points: baseline (T0), after 2, 4, 6 and 8 weeks of use (Tw). The reduction of skin wrinkle and roughness was calculated as in

Reduction $(\%)=\left(\mathrm{SELS}_{\mathrm{Tw}} / \mathrm{SELS}_{\mathrm{T} 0}\right)$ test formula / $\left(\mathrm{SELS}_{\mathrm{Tw}} / \mathrm{SELS}_{\mathrm{T} 0}\right)$ vehicle $\times 100 \%$

\subsection{Statistical Analysis}

All experiments were repeated at least three times with different batches of cells. All data are presented as mean \pm S.D. with GraphPad Prism. Data were evaluated statistically using Student's t-test, and the statistical significance was set at $\mathrm{p}<0.05$. For transcriptome analysis, statistical significance was set at $\mathrm{p}<0.05$ and fold change $\geq 1.5$. 


\section{Results and Discussion}

\subsection{PTC Improves the Proliferation of HDF-a Fibroblasts and NHEK Keratinocytes}

Cellular senescence plays a vital role in regulating cellular aging both in vitro and in vivo. The aging cells are identified with a distinct phenotype, which includes flat morphology, bulged cell size, slowed proliferation rate and changes in protein and gene level [24, 25].

MTT results showed that the highest cell viability rate were $155.3 \%$ after $0.25 \%$ of PTC treatment for $72 \mathrm{hrs}$ on HDF-a cells (Figure 1a), and for 24 and $48 \mathrm{hrs}$ PTC at $0.125 \%$ to $0.5 \%$ concentrations still significantly improved the cell viability. On NHEK cells, the cell viability was maximum induced to $239.4 \%$ after $0.016 \%$ of PTC treatment for 72 hrs (Figure $1 \mathrm{~b}$ ). It verified the proliferation efficacy of PTC in epidermal keratinocytes and dermal fibroblasts.

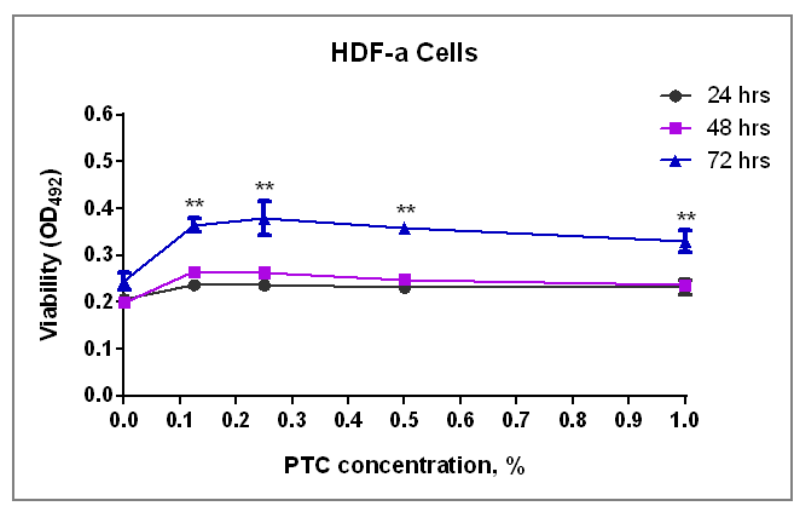

(a)

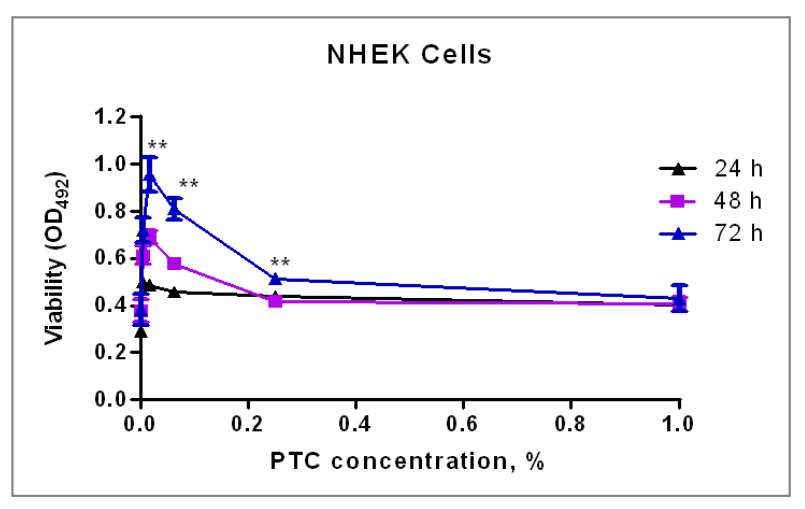

(b)

Figure 1. Proliferation effect of PTC on and fibroblast (a) and keratinocyte (b) cells.

\subsection{PTC Affected Lots of Genes Involved in Cell Senescence}

The gene expression changes with age in various human tissues including skin [26]. Previous studies reported that during replicative senescence, dermal fibroblasts presented a deregulation of genes involved in various skin cellular processes such as inflammatory response, cell cycle regulation and signal transduction [27-29].
Focusing on the key genes related to cell cycle, secretory features and macromolecular damage [30-32], we identified the anti-aging mechanism regulated by PTC. Total 935 genes were changed after $0.5 \%$ PTC treatment for $24 \mathrm{hrs}, 439$ upregulated and 496 downregulated. First significant variations in cell-cycle arrest were identified in table 1 , such as cyclins, cyclin dependent kinases (CDKs) and $\mathrm{CDK}$ inhibitors (CDKN1A and CDKN2A). The proteins encoded by these genes are classified as the key protein required for cell cycle progression in G0/G1 to S phase.

Table 1. Modulated genes in cell-cycle arrest after PTC treatment.

\begin{tabular}{lll}
\hline Gene symbol & Description & Fold change \\
\hline CCNA2 & Cyclin A2 & 1.24 \\
CCNB1 & Cyclin B1 & 1.55 \\
CCND1 & Cyclin D1 & 1.68 \\
CDK2 & Cyclin dependent kinase 2 & 1.21 \\
CDK4 & Cyclin dependent kinase 4 & 1.51 \\
CDKN1A & Cyclin-dependent kinase inhibitor 1A & 0.66 \\
CDKN2A & (p21, Cip1) & 0.89 \\
RB1 & Cyclin-dependent kinase inhibitor 2A & 0.82 \\
TP53 & Tumoblinostoma 1 & 0.63 \\
\hline
\end{tabular}

Furthermore, PTC can improve the expression of key senescence-associated insoluble factors in figure 2, such as collagens (COL5A3, COL6A1, COL7A1), elastin (ELN), mucooplysaccharide (BGN, CD44), laminins (LAMA1, LAMC5), intergrins (ITGA2, ITGB1), epiregulin (EREG), intercellular adhesion molecule 5 (ICAM5), fibrillin 1 (FBN1), fibronectin (FN), procollagen-lysine (PLOD1) and tenascin C (TNC) to strengthen cell function and adhesion [33-36]. All the proteins encoded by these genes are downregulated in senescent cells to strengthen skin aging.

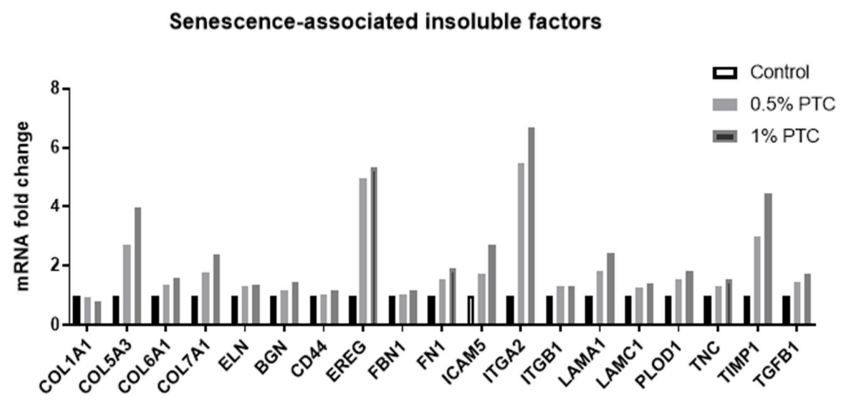

Figure 2. The $m R N A$ expression changes of secretory features in fibroblast treated with PTC.

\subsection{PTC Induced the Synthesis of ECM Proteins}

Dermal fibroblasts are thought to be responsible for synthesising the three major groups of dermal ECM proteins including fibrillar collagens, elastic fibres and proteoglycans $[2,9]$. According to the gene expression results, the content of ECM proteins was analyzed further.

As shown in figure 3, PTC treated cells synthesized more amount of type I collagen and type III collagen than untreated control cells after $48 \mathrm{hrs}$. 


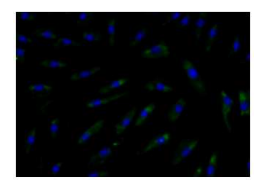

(a)

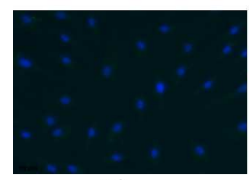

(d)

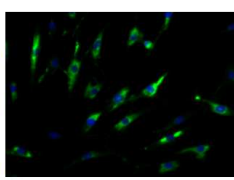

(b)

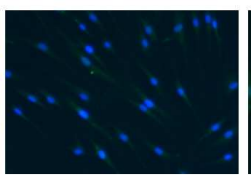

(e)

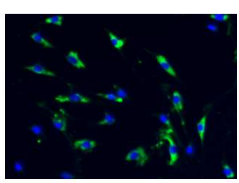

(c)

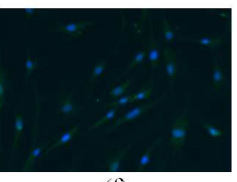

(f)
Figure 3. PTC increases the expression of collagens on HDF-a (100X). (a-c): type I collagen, control w/o FBS, PTC 0.25\%, PTC 0.5\%; (d-f): type III collagen, control w/o FBS, PTC $0.25 \%$, PTC $0.5 \%$.

We also tested the extracellular content of other ECM proteins for $48 \mathrm{hrs}$ on HDF-a and NHEK cells treated with PTC $(0.25 \%$ to $1 \%)$. As listed in table 2 , when cells were treated with $0.25 \%$ PTC for $48 \mathrm{hrs}$, the extracellular content of type I collagen was increased from 225.39 to $327.39 \mathrm{ng} / \mathrm{ml}$ and type III collagen increased from 0.66 to $1.31 \mathrm{ng} / \mathrm{ml}$ with a maximum growth rate of 145.2 and $198.5 \%$ respectively. The content of fibronectin was also increased from 400.31 to $740.20 \mathrm{ng} / \mathrm{ml}$. Focusing on the ECM proteins secreted by keratinocytes, the extracellular amount of type IV and type VII collagen was regulated from 0.27 to $1.06 \mathrm{ng} / \mathrm{ml}$ and from 3.70 to $9.81 \mathrm{ng} / \mathrm{ml}$ by $1 \%$ PTC treated for $48 \mathrm{hrs}$, and the maximum rate of growth of intergrin $\beta 1$ was moderately upregulated to $176.6 \%$ compared to untreated cells, while laminin 5 was not changed with data not shown.

For the TGF- $\beta 1$, a cytokine that contributes primarily to the biosynthesis of collagen associated with cutaneous aging [37], was upregulated in both HDF-a cells and NHEK cells after PTC treatment for $48 \mathrm{hrs}$. The growth rate reached to $331.4 \%$ and $175.6 \%$ respectively. These results confirmed the fold change of gene expression which related to the pathway and cell functions in skin aging, from dermis to epidermis, especially the dermal-epidermal junction.

Table 2. The expression of ECM proteins after PTC treatment.

\begin{tabular}{lllll}
\hline Protein name, $\mathbf{n g} / \mathbf{m l}$ & Cells & Control & PTC 0.25\% & PTC 0.5\% \\
\hline Type I collagen & HDF-a & 225.39 & 327.39 & 304.19 \\
Type III collagen & HDF-a & 0.66 & 1.31 & 1.22 \\
Fibronectin & HDF-a & 400.31 & 740.20 & 668.82 \\
Type IV collagen & NHEK & 0.27 & 0.54 & 0.62 \\
Type VII collagen & NHEK & 3.70 & 6.26 & 6.51 \\
Intergrin $\beta 1$ & NHEK & 8.86 & 8.97 & 9.06 \\
TGF- $\beta 1$ & HDF-a & 0.035 & 0.136 & 0.70 \\
TGF- $\beta 1$ & NHEK & 0.307 & 0.403 & 0.152 \\
\hline
\end{tabular}

\subsection{PTC Reduced the Wrinkles and Roughness in Human Skin}

As a person ages, proliferation of cells in the basal layer reduces. The epidermis then becomes thinner, the contact surface area between dermis and epidermis decreases, and demal epidermal junction flatting, resulting in skin wrinkles and roughness [38]. Furthermore, the clinical improvement of PTC (left side) was compared to the placebo (right side) on human skin around eyes (Figure 4). When applied with formulas contained 5\% PTC for 8 weeks, the surface evaluation of wrinkles (SEw) and surface evaluation of roughness (SEsm) was reduced from $100 \%$ to $80.6 \%$ and $86.3 \%$ respectively. Macrophotography of the left side of the face applied of PTC for 6 weeks also showed a reduction in length and width of wrinkles compared to placebo.

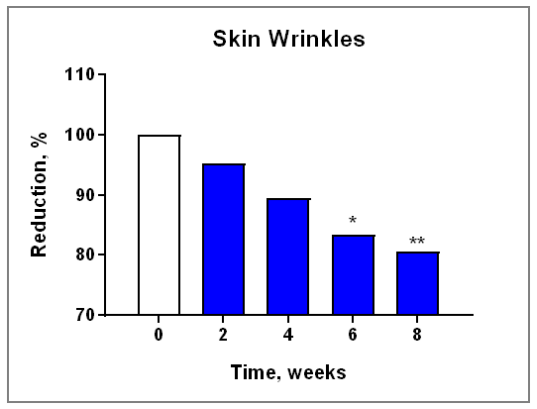

(a)

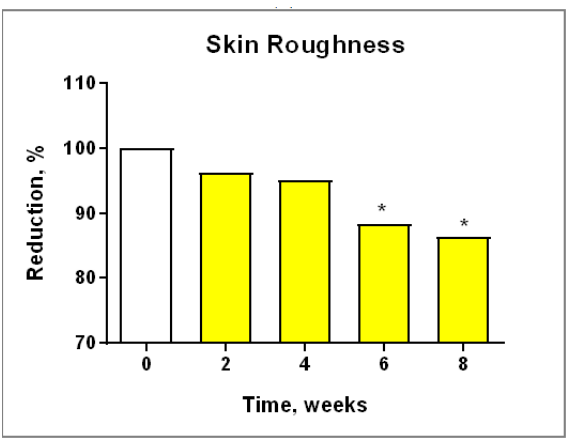

(b)
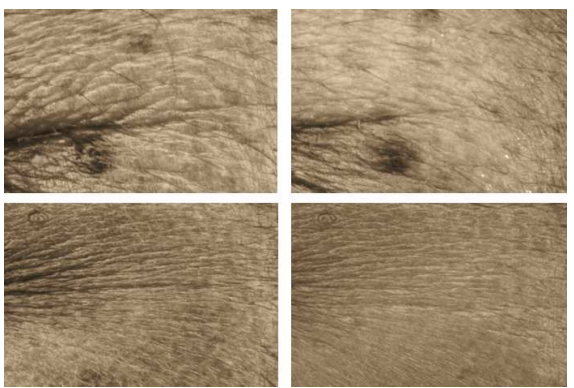

(c)

Figure 4. PTC improved the skin wrinkles and roughness. (a-b) The reduction of skin wrinkles and roughness. (c) PTC before and after use of two subjects (above: Subject \#10, 47 old-years; below: Sebject \#15, 32 old-years). 


\section{Conclusions}

Cellular senescence is a cell state implicated in various physiological processes and a wide spectrum of age-related diseases. Dermis thickness decreases with age; thinning is accompanied by a decrease in both vascularity and cellularity. There is a decrease in the number of fibroblasts, the amount of glycosaminoglycans, collagens and elastin, as well as a loss of molecular integrity and tight junction [39]. PTC, a palmitoyl oligopeptide complex, can stimulate the proliferation and strengthen the synthesis of ECM proteins in dermal fibroblast, to against cell senescence.

In senescent cells, the CDK2 inhibitor CDKN1A, 1C and CDK4/6 inhibitor CDKN2A were accumulated to result in activation of retinoblastoma (RB) and p53 family proteins, inhibition of E2F transactivation, and consequent cell-cycle arrest [32]. PTC prevented the cell-cycle arrest by upregulating the mRNA expression of CCNA2, CCNB1, CCND1, CDK2, CDK4, and downregulating the mRNA expression of CDKN1A, 1C, 2A, RB family members and p53 family members, to promote cell proliferation.

For the other certain physiological processes related to cellular senescence, secretory features, PTC not only affected the mRNA level of key genes, such as COL5A3, COL6A1, COL7A1, ELN, BGN, CD44, LAMA1, LAMC5, ITGA2, ITGB1, EREG, ICAM5, FBN1, FN, TNC and TIMP1, but also regulated the protein synthesis, including four type collagens, fibronectin, integrin $\beta 1$ as well.

Transforming growth factor- $\beta 1$ (TGF $\beta 1$ ) acted as an important modulator of cell growth, inflammation, collagen synthesis and apoptosis. Many of the dermal ECM-related genes that are downregulated in aged human skin are regulated by the TGF- $\beta$ pathway [40-42]. Focusing on the TGF- $\beta 1$ pathway, PTC not only enhanced the TGF- $\beta 1$ expression to promote the synthesis of collagens, elastin, fibronectin, and intergrins in fibroblasts, but also promoted the synthesis of TGF- $\beta 1$ in keratinocytes to act as an inducer of cell renewal. The molecular pathway maybe targets the TGF- $\beta 1$ in both epidermis and dermis.

Further, PTC certified its anti-aging effect on the clinical study targeting facial wrinkles and roughness for 8 weeks. The surface evaluation of wrinkles (SEw) and surface evaluation of roughness (SEsm) was significantly reduced at week 6 compared to placebo. All data suggest PTC had a 3D theory against skin aging: from epidermal keratinocyte to dermal fibroblast, especially containing the structural proteins of DEJ.

\section{Acknowledgements}

Research and development was done by Shanghai JAKA Biotech Co. Ltd.

\section{References}

[1] Rinnerthaler M, Streubel M K and Bischof J (2015). Skin aging, gene expression and calcium. Experimental Gerontology, 68: $59-65$.
[2] Naylor EC, Watson REB and Sherratt MJ (2011). Molecular aspects of skin ageing. Maturitas, 69 (3): 249-256.

[3] Kohl E, Steinbauer J and Landthaler M (2011). Skin ageing. Journal of the European Academy of Dermatology and Venereology, 25 (8): 12.

[4] Zhang S and Duan E (2018). Fighting against Skin aging: The way from bench to bedside. Cell Transplantation, 27 (2): 096368971772575 .

[5] Lin'kova NS, Drobintseva AO and Orlova OA (2016). Peptide regulation of skin fibroblast functions during their aging in vitro. Bulletin of Experimental Biology and Medicine, 161 (1): 175-178.

[6] Shi Yue-ying, Qin De-zhi and Meng Fan-ling (2016). Efficacy of peptides in cosmetics. China cosmetics, 7: 80-82.

[7] Mcfarland GA and Holliday R (1994). Retardation of the senescence of cultured human diploid fibroblasts by carnosine. Experimental Cell Research, 212 (2): 170-175.

[8] Quan T, Wang F and Shao Y (2013). Enhancing structural support of the dermal microenvironment activates fibroblasts, endothelial cells, and keratinocytes in aged human skin in vivo. Journal of Investigative Dermatology, 133 (3): 658-667.

[9] Fisher GJ, Shao Y and He T (2016). Reduction of fibroblast size/mechanical force down-regulates TGF- $\beta$ type II receptor: implications for human skin aging. Aging Cell, 15 (1): 67-76.

[10] Xie HF, Tang H and Ji LI (2006). The expression of FoxO3a and BubR1 in the process of skin intrinsic aging. Journal of Clinical Dermatology, 35 (3): 149-151.

[11] de Araújo R, Lôbo M and Trindade K (2019). Fibroblast growth factors: a controlling mechanism of skin aging. Skin Pharmacol Physiol, 32 (5): 275-282.

[12] Celebibirand ED, Karoglu ET and Doldurballi F (2016). Mammalian target of rapamycin (mTOR), aging, neuroscience, and their association with aging-related diseases. Molecules to Medicine with Mtor, 185-203.

[13] Varma SR, Sivaprakasam TO and Abheepsa M (2016). Protective effects of triphala on dermal fibroblasts and human keratinocytes. PLoS One, 11 (1): e0145921.

[14] Chun-Shan Liu, Tae-Gyu Nam and Min-Woo Han (2013). Protective effect of detoxified rhus verniciflua stokes on human keratinocytes and dermal fibroblasts against oxidative stress and identification of the bioactive phenolics. Biosci Biotechnol Biochem, 77 (8): 1682-1688.

[15] Cho BA, Yoo SK and Seo JS (2018). Signatures of photo-aging and intrinsic aging in skin were revealed by transcriptome network analysis. Aging, 10 (7): 1609-1626.

[16] Wei Y, Li-Li Z and Li Y (2013). Transcriptome analysis of skin photoaging in chinese females reveals the involvement of skin homeostasis and metabolic changes. PLoS One, 8 (4): e61946.

[17] Breitenbach JS, Rinnerthaler M and Trost A (2015). Transcriptome and ultrastructural changes in dystrophic Epidermolysis bullosa resemble skin aging. Aging, 7 (6): 389-411.

[18] Scheerder ID, Buyzere MD and Delanghe J (1989). Autoimmune response to collagen type I, III, and IV in endocarditis evaluated by ELISA technique. Journal of Molecular and Cellular Cardiology, 21 (supp-S4): S23. 
[19] Yang H, Li J and Wang Y (2014). Role of CD14 and TLR4 in type I, type III collagen expression, synthesis and secretion in LPS-induced normal human skin fibroblasts. International Journal of Clinical \& Experimental Medicine, 8 (2): 2429-2434

[20] Marionnet C, Pierrard Cécile and Vioux-Chagnoleau C (2006). Interactions between fibroblasts and keratinocytes in morphogenesis of dermal epidermal junction in a model of reconstructed skin. Journal of investigative dermatology, 126 (5): 971-979.

[21] Newton VL, Bradley RS and Seroul P (2017). Novel approaches to characterize age-related remodelling of the dermal epidermal junction in $2 \mathrm{D}, 3 \mathrm{D}$ and in vivo. Skin Research and Technology, 23 (2): 131-148.

[22] BA Khan (2012). Visio Scan ${ }^{\circledR}$ VC98, Corneometer MPA 5 and Tewameter MPA 5. African Journal of Pharmacy \& Pharmacology, 6 (3): 225-227.

[23] Pillai R, Redmond M and Röding J (2005). Anti-wrinkle therapy: significant new findings in the non-invasive cosmetic treatment of skin wrinkles with beta-glucan. International Journal of Cosmetic Science, 27 (5): 292-292.

[24] Laura Rojas Lorz, Byong Chul Yoo and Mi-Yeon Kim (2019). Anti-wrinkling and anti-melanogenic effect of pradosia mutisii methanol extract. Int J Mol Sci., 20 (5): 1043.

[25] Dimri GP, Lee XH and Basile G (1995). A biomarker that identifies senescent human-cells in culture and in aging skin in-vivo. Proceedings of the National Academy of Sciences of the United States of America, 92 (20): 9363-9367.

[26] Glass D, Viñuela A and Davies MN (2013). Ramasamy A, Gene expression changes with age in skin, adipose tissue, blood and brain. Genome Biology, 14 (7): R75.

[27] Shelton DN, Chang E and Whittier PS (1999). Microarray analysis of replicative senescence. Current Biology, 9 (17): 939-945.

[28] Yoon IK, Kim HK and Kim YK (2004). Exploration of replicative senescence-associated genes in human dermal fibroblasts by cDNA microarray technology. Experimental Gerontology, 39 (9): 1369-1378.

[29] Stéphanie D, Philippe C and Marion W (2011). DNA macroarray study of skin aging-related genes expression modulation by antioxidant plant extracts on a replicative senescence model of human dermal fibroblasts. Phytotherapy Research, 25 (5): 686-693.
[30] Beausejour C. M (2003). Reversal of human cellular senescence: roles of the p53 and p16 pathways. EMBO (European Molecular Biology Organization) Journal, 22 (16): 4212-4222.

[31] Rodier F and Campisi J (2011). Four faces of cellular senescence. The Journal of Cell Biology, 192 (4): 547-556.

[32] Vassilis G, Peter D. A and Andrea A (2019). Cellular senescence: defining a path forward [J]. Cell, 179: 813-827.

[33] Qin G, Luo M and Chen J (2016). Reciprocal activation between MMP-8 and TGF- $\beta 1$ stimulates EMT and malignant progression of hepatocellular carcinoma. Cancer Letters, 374 (1): 85-95.

[34] Caterina Longo (2016). Well-aging: Early detection of skin aging signs. Dermatol Clin., 34 (4): 513-518.

[35] Coppé Jean-Philippe, Desprez PY andKrtolica A (2010). The senescence-associated secretory phenotype: the dark side of tumor suppression. Annual Review of Pathology Mechanisms of Disease, 5 (1): 99-118.

[36] Kuilman T and Peeper DS (2009). Senescence-messaging secretome: SMS-ing cellular stress. Nature Reviews Cancer, 9 (2): 81-94.

[37] Han KH, Choi HR and Won CH (2005). Alteration of the TGF- $\beta$ /SMAD pathway in intrinsically and UV-induced skin aging. Mechanisms of Ageing \& Development, 126 (5): 560-567.

[38] Makrantonaki E, Zouboulis CC and William J (2007). Characteristics and pathomechanisms of endogenously aged skin. Dermatology, 214 (4): 352-360.

[39] Farage MA, Miller KW and Elsner P (2013). Characteristics of the aging skin. Advances in Wound Care, 2 (1): 5-10.

[40] Annes JP (2003). Making sense of latent TGFbeta activation. Journal of Cell Science, 116 (2): 217-224.

[41] He T, Quan T, Shao Y, Voorhees JJ, Fisher GJ (2014). Oxidative exposure impairs tgf-beta pathway via reduction of type ii receptor and smad3 in human skin fibroblasts. Age (Dordr), 36: 9623.

[42] Shin JW, Kwon SH and Choi JY (2019). Molecular mechanisms of dermal aging and antiaging approaches. International journal of molecular sciences, 20 (9): 2126. 